Monatsschr Kinderheilkd 2018 • 166:910-911 https://doi.org/10.1007/s00112-018-0570-7 Online publiziert: 12 . September 2018 (c) Der/die Autor(en) 2018

\section{Redaktion}

R. Berner, Dresden

B. Koletzko, München

A.C. Muntau, Hamburg

W. Sperl, Salzburg

CrossMark

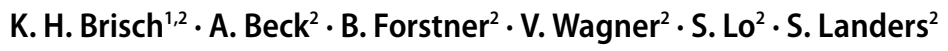

${ }^{1}$ Institut für Early Life Care, Paracelsus Medizinische Privatuniversität PMU Salzburg, Salzburg, Österreich ${ }^{2}$ Pädiatrische Psychosomatik und Psychotherapie, Dr. von Haunersches Kinderspital, LMU München, München, Deutschland

\title{
Resignation/Dissoziation des Säuglings statt gelungener Selbstregulation
}

Einschlafen verbunden ist, was sich als Verhaltensmuster einprägt, nach kurzer Zeit umsetzt und dabei hilft, eigenständig zur Ruhe zu finden. (S. 80)

Bindt C, Schulte-Markwort M (2017) Schreien und persistierende Unruhe im Säuglings- und Kleinkindalter. Monatsschr Kinderheilkd 165: 73-85. https://doi.org/10.1007/s00112-0160229-1

\section{Erwiderung}

Bindt C (2018) Exzessives Säuglingsschreien, persistierende Unruhe und

Bindungsentwicklung: Plädoyer für einen erweiterten Blick. Monatsschr Kinderheilkd. https://doi.org/10.1007/s00112-018-0572-5

Mit großem Interesse haben wir den CME-zertifizierten Beitrag „Schreien und persistierende Unruhe im Säuglings- und Kleinkindalter" zur Kenntnis genommen und möchten hiermit zu den von den Autoren getroffenen Aussagen Stellung nehmen.

Wir beziehen uns insbesondere auf den Abschnitt „Verhaltensbezogene Interventionen".

Die Autoren schreiben hier:

Man kann dazu ermutigen, das Kind auch schreiend ins Bett zu legen und abzuwarten, bis es nach 15-30 min, oft abrupt, einschläft. Da diese Zeitspanne Eltern endlos erscheint, empfiehlt sich der Blick auf die Uhr. Bleibt das Kind unruhig, sollte nach kurzem Stillen oder Füttern ein erneuter Versuch gestartet werden. Das Kind lernt dabei, dass das Hochnehmen mit Aktivität und das Ablegen mit Ruhezeiten und dem

Ein weiterer Leserbrief zu https://doi.org/10. 1007/s00112-016-0229-1 ist unter https://doi. org/10.1007/s00112-018-0571-6zu finden.
Sie nennen in diesem Zusammenhang jedoch keine Studie, die diese Methode, v. a. das angegebene Zeitintervall, wissenschaftlich belegen könnte. Dies entspricht in keiner Weise dem Anspruch einer evidenzbasierten Medizin, dem die Autoren im Rahmen eines CME-zertifizierten Artikels jedoch gerecht werden sollten. Die Minutenzahl erscheint willkürlich gewählt. Man bedenke, dass $30 \mathrm{~min}$ eine $100 \%$ ige Verlängerung von $15 \mathrm{~min}$ bedeuten. Der Erwachsene hat zwar anhand einer Uhr die Kontrolle über eine solche Zeitdauer, ein Säugling verfügt jedoch noch nicht über ein solches Zeitgefühl und scheint dieser Intervention hilflos ausgeliefert.

Aus bindungstheoretischer und entwicklungspsychologischer Sicht stellt das Schreien ein evolutionäres Signal dar, das die Nähe zu den Eltern bzw. seiner Bindungsperson aufrechterhalten oder wiederherstellen soll und für den Säugling überlebensnotwendig ist (u.a. [3, 6, 9]). Das feinfühlige, prompte Eingehen auf die kindlichen Signale (z. B. insbesondere auf das Schreien) vermittelt dem Säugling die Sicherheit, dass er auf die Anwesenheit der Eltern auch in Zeiten innerer Not vertrauen kann (u.a. [1]). Entgegen der in früheren Zeiten vorherrschenden Meinung, dass ein Säugling umso mehr weine, je mehr man darauf einginge [7], belegten Ainsworth et al. [2] genau das Gegenteil: Mütter, die prompt und ein- fühlsam auf das Weinen ihrer Kleinkinder schon in den ersten Lebenswochen reagierten, hatten später Kleinkinder, die weniger weinten.

Tatsächlich ist die Studienlage zum Thema „Schreienlassen“ oder "Nichtschreien-Lassen" heute nicht so eindeutig, wie Ainsworth et al. [2] feststellten. So verglichen beispielsweise St. JamesRoberts et al. [8] Eltern, die ihr Kind $80 \%$ der Zeit zwischen 8 und 20 Uhr trugen, es regelmäßig stillten und schnell auf das Schreien reagierten, mit einer Gruppe von Eltern, die - auch in Zeiten des Schreiens - $50 \%$ weniger Körperkontakt mit ihren Säuglingen hatten als die der ersten Gruppe und früher abstillten. Die Ergebnisse zeigten, dass die Kinder der ersten Gruppe im Alter von 2 und 5 Wochen $50 \%$ weniger schrien als die Kinder der zweiten Gruppe. Gleichzeitig wurde jedoch auch deutlich, dass die Kinder der ersten Gruppe im Alter von 12 Wochen nachts häufiger erwachten und schrien als die Kinder der zweiten Gruppe. Schreien, das durch die Eltern nicht $\mathrm{zu}$ stillen ist („unsoothable crying"), trat in beiden Gruppen gleich häufig auf.

$\mathrm{Ob}$ „Schreienlassen“ nun zu weniger nächtlichem Erwachen bzw. Schreien des Säuglings führt oder nicht, fest steht: Der Säugling befindet sich bei andauerndem Schreien außerhalb seines Stresstoleranzfensters [12, 15], also außerhalb des Bereichs, in dem er seinen Stress in diesem Alter selbst regulieren kann. Dies zeigt sich auch im Anstieg des Kortisollevels des Säuglings, der mit der Länge des Schreiens korreliert [16]. Erfährt 
der Säugling wiederholt keine Antwort auf das Schreien, erlebt er wiederkehrende Enttäuschungen und wird dieses Verhalten - Schreien bei erlebter Belastung/Bedrohung - trotz der inneren Übererregung aufgeben [13]. Das Kind lernt, dass es mit seinem Schreien nichts bewirken kann und in einem hilflosen Zustand allein gelassen wird [6, 10]. So zeigt beispielsweise die Studie von Middlemiss et al. [11], dass im Rahmen eines 5-tägigen Schlaftrainings die Stresslevel der schreienden Säuglinge und auch das Stresserleben der Mütter sehr hoch waren. Nach 3 Tagen hatten die Säuglinge zwar aufgehört $\mathrm{zu}$ schreien, ihre Stresslevel waren jedoch unverändert hoch - lediglich der Stress der Mütter ließ nach.

Abruptes Einschlafen scheint folglich eher ein Zeichen der Resignation bzw. Dissoziation zu sein als das einer gelungenen Selbstregulation des Säuglings.

Ein bedeutender Punkt neben der feinfühligen Reaktion auf das Schreien, den auch die Autoren nennen, ist sicher die Unterstützung des Säuglings in der Entwicklung seiner Selbstwirksamkeit/ Selbstregulationsfähigkeit. Hier empfehlen beispielsweise auch Wolke et al. [17], nicht bei jeder negativen Äußerung des Kindes - wie Jammern oder Nörgeln sofort zu reagieren oder gar übermäßig $\mathrm{zu}$ stimulieren, sondern ca. 1-2 min ab $\mathrm{zu}$ warten, um die Regulationsfähigkeit des Kindes zu verbessern. Dies steht jedoch unserer Meinung nach in keinem Verhältnis zu dem von den Autoren vorgeschlagenen Zeitraum von 15-30 min. Eine Studie zeigt außerdem, dass insbesondere die koregulierende Reaktion der Mutter auf den Säugling in Stresssituationen die Regulationsfähigkeiten des Kindes im Schreien und im Schlafen erhöht. Feinfühlige Interaktionen beispielsweise im Spiel hatte keinen so großen Einfluss auf diese [14].

Auf Grundlage der Bindungstheorie empfehlen wir deshalb allen (werdenden) Eltern, auf die Signale des Kindes prompt und feinfühlig einzugehen und es auf keinen Fall in einem Zustandlauten Weinens oder Schreiens sich selbst zu überlassen. Damit meinen wir nicht eine übervorsichtige Reaktion der Eltern auf jedes Signal der Unzufriedenheit, sondern eine
- auf die individuelle Regulationsfähigkeit des Kindes - abgestimmte Antwort, die die Weinkurve des Kindes (Quengeln, Jammern, lautes Weinen, Schreien, panisches Schreien) und die individuelle Entwicklungsstufe des Kindes (Wie viel Regulation schafft das Kind allein? Wann braucht es die Hilfe der Eltern?) beachtet.

Herauszufinden, was der schreiende Säugling innerlich erlebt, welches Bedürfnis und welcher Affekt den Säugling so sehr stressen, dass er außerhalb seines Stresstoleranzfensters gerät, und angemessen sowie differenziert darauf $\mathrm{zu}$ reagieren, sowie ihm $\mathrm{zu}$ helfen, sich $\mathrm{zu}$ regulieren, erscheint uns der Dreh- und Angelpunkt. Eltern hilft dabei die Fähigkeit, ihrem Kind einerseits etwas zutrauen und zumuten zu können, andererseits sich gut in das Kind und seine Bedürfnisse hineinversetzen sowie seine Gefühle und Absichten erkennen zu können. Im Ausbau dieser Fähigkeiten sollten die Eltern unterstützt werden, wie dies etwa in verschiedenen Präventionsprogrammen zur Elternschulung (z. B. SAFE ${ }^{\circledR}[\mathrm{Si}-$ chere Ausbildung für Eltern], STEEP ${ }^{\mathrm{m}}$ [Steps Toward Effective, Enjoyable Parenting] etc.) und durch Interventionen der Familienhebammen im Rahmen der Frühen Hilfen bereits umgesetzt wird. Gleichzeitig besteht die Notwendigkeit, der Mutter oder Bezugsperson in Situationen von erlebter Hilflosigkeit (unstillbares Weinen des Säuglings) Unterstützung zukommen zu lassen; dies kann von der Verbesserung des sozialen Unterstützungssystems, der Anleitung zur Selbstberuhigung bis hin $\mathrm{zu}$ therapeutischer Unterstützung reichen, etwa bei postpartaler Depression oder bei nichtgelösten traumatischen Erfahrungen.

\section{Korrespondenzadresse}

\section{PD Dr. med. K. H. Brisch}

Institut für Early Life Care, Paracelsus

Medizinische Privatuniversität PMU Salzburg

Salzburg, Österreich

Karl-Heinz.Brisch@med.uni-muenchen.de

Interessenkonflikt. K.H. Brisch, A. Beck, B. Forstner, V. Wagner, S. Lo und S. Landers geben an, dass kein Interessenkonflikt besteht.

Open Access. Dieser Artikel wird unter der Creative Commons Namensnennung 4.0 International Lizenz (http://creativecommons.org/licenses/by/4.0/deed. de) veröffentlicht, welche die Nutzung, Vervielfältigung, Bearbeitung, Verbreitung und Wiedergabe in jeglichem Medium und Format erlaubt, sofern Sie den/die ursprünglichen Autor(en) und die Quelle ordnungsgemäßnennen, einen Link zur Creative Commons Lizenz beifügen und angeben, ob Änderungen vorgenommen wurden.

\section{Literatur}

1. Ainsworth MDS (1974) The development of infantmother attachment. A final report of the office of child development. Office of Child Development, Washington D.C

2. Ainsworth MDS, Bell SM, Stayton DJ (1971) Individual differences in strange-situational behaviour of one-year-olds. In: Schafer HR (Hrsg) The Origins of Human Social Relations. Academic Press, London, S17-42

3. Bell SM, Ainsworth MDS (1972) Infant crying and maternal responsiveness. Child Dev 43:1171-1190

4. Bindt C, Schulte-Markwort M (2017) Schreien und persistierende Unruhe im Säuglings - und Kleinkindalter (CME-Fortbildung). Monatsschr Kinderheilkd 165:73-85

5. Bowlby J (1975) Bindung. Eine Analyse der MutterKind-Beziehung. Fischer, Frankfurt/M.

6. Bowlby J (1969/2006) Bindung und Verlust 1: Bindung. Reinhardt, München

7. Haarer J (1934) Die deutsche Mutter und ihr erstes Kind Bd. 2002. Carl-Gerber Verlag, München, S 29-36

8. James-Roberts $S$ et al (2006) Infant crying and sleeping in London, Copenhagen and when parents adopt a „proximal “ form of care. Pediatrics 117(6):1146-1155

9. Ludington-Hoe S, Cong X, Hashemi F (2002) Infant crying: nature, physiologic consequences, and select interventions. Neonatal network 21(2):29-36

10. Main M, Kaplan N, Cassidy J (1985) Security in infancy, childhood and adulthood: a move to the level of representation. In: Bretherton I, Waters E ( (Hrsg) $<\mathrm{i}>$ Growing points of attachment theory and research, 50. University of Chicago Press, Chicago

11. Middlemiss W, Granger DA, Goldberg WA, Nathans L (2012) Asynchrony of mother - infant hypothalamic - pituitary - adrenal axis activity following extinction of infant crying responses induced during the transition to sleep. Early Hum Dev 88(4):232

12. Ogden P, Minton K, Pain C (2006) Trauma and the body: a sensorimotor approach to psychotherapy. WW Norton, New York

13. Perry BD, Pollard RA, Blakley TL, BakerWL, Vigilante D (1995) Childhood trauma, the neurobiology of adaptation, and "use-dependent" development of the brain: How "states" become „traits". Infant Ment Health J 16(4):271-291

14. Richter N, Reck C (2013) Positive maternal interaction behavior moderates the relation between maternal anxiety and infant regulatory problems. Infant BehavDev 36(4):498-506

15. Siegel DJ (1999) The developing mind. Guilford, New York

16. Taylor A, Fisk NM, Glover V (2000) Mode of delivery and subsequent stress response. Lancet 355(9198): 120

17. Wolke D, Gray P, Meyer R (1994) Excessive Infant Crying: A Controlled Study of Mothers Helping Mothers. Pediatrics 94(3):322-332 